Z Herz- Thorax- Gefäßchir 2018 · 32:286-299 https://doi.org/10.1007/s00398-018-0246-6 Online publiziert: 12. Juni 2018

(c) Der/die Autor(en) 2018

CrossMark

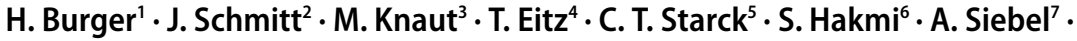

A. Böning ${ }^{8}$

${ }^{1}$ Abteilung für Herzchirurgie, Kerckhoff-Klinik GmbH, Bad Nauheim, Deutschland

${ }^{2}$ Klinik für Kardiologie und Angiologie, Universitätsklinikum Gießen und Marburg (UKGM), Gießen, Deutschland

${ }^{3}$ Klinik für Herzchirurgie, Herzzentrum Dresden, Universitätsklinik, Technische Universität Dresden, Dresden, Deutschland

${ }^{4}$ Klinik für Thorax- und Kardiovaskularchirurgie, Herz- und Diabeteszentrum Nordrhein-Westfalen, Bad Oeynhausen, Deutschland

${ }^{5}$ Klinik für Herz-, Thorax- und Gefäßchirurgie, Deutsches Herzzentrum Berlin, Berlin, Deutschland

${ }^{6}$ Klinik für Herz- und Gefäßchirurgie, Universitäres Herzzentrum (UHZ), Hamburg, Deutschland

${ }^{7}$ Klinik für Kardiochirurgie, Herz- und Gefäß-Klinik Bad Neustadt, Bad Neustadt an der Saale, Deutschland ${ }^{8}$ Klinik für Herz-, Kinderherz-, und Gefäßchirurgie, Universitätsklinikum Gießen und Marburg (UKGM), Gießen, Deutschland

\title{
Einsatz des tragbaren Kardioverter-Defibrillators nach kardiochirurgischen Eingriffen
}

\section{Positionspapier der AG Herzrhythmusstörungen der Deutschen Gesellschaft für Thorax-, Herz- und Gefäßchirurgie}

\section{Inhaltsverzeichnis}

1. Präambel

2. Einleitung und Hintergrund

3. Aufbau und Funktionsweise des tragbaren Kardioverter-Defibrillators

4. Bisherige klinische Erfahrungen mit dem WCD

5. Indikationen zum Einsatz des WCDs im Rahmen kardiochirurgischer Therapien

5.1. Primärprophylaktische Indikationen

5.1.1. Koronare Herzerkrankung, ischämische Kardiomyopathie, Myokardinfarkt und Myokardrevaskularisation

5.1.2. Dilatative Kardiomyopathien, nichtischämische Kardiomyopathien und Myokarditiden

5.1.3. Valvuläre Kardiomyopathien
5.2. Sekundärprophylaktische Indikation nach hämodynamisch relevanten Rhythmusstörungen

5.3. Kontraindikation zur ICD-Implantation bei bestehender ICDIndikation

5.4. WCD-Indikation im Rahmen von ICD-Explantationen

5.5. Sonderfälle

6. Kosteneffektivität der temporären WCD-Therapie

7. Implementierung eines WCD-Programms in den klinischen Alltag

8. Schlussfolgerung

\section{Präambel}

Dies ist ein Positionspapier der Arbeitsgruppe Herzrhythmusstörungen der Deutschen Gesellschaft für Thorax-, Herz- und Gefäßchirurgie (DGTHG). Es spiegelt den aktuellen Stand des medizinischen Wissens zur temporären
Versorgung von Hochrisikopatienten des plötzlichen Herztods (PHT) mit dem tragbaren Kardioverter-Defibrillator (,wearable cardioverter defibrillator“, WCD) wider und soll Ärzten eine praxisgerechte Hilfestellung für dessen Indikationsstellung und den klinischen Einsatz geben. Diese Empfehlungen ersetzen allerdings nicht die individuelle und der klinischen Situation angepasste ärztliche Evaluation des einzelnen Patienten.

\section{Einleitung und Hintergrund}

Der implantierbare Kardioverter-Defibrillator (ICD) ist seit über 30 Jahren integraler Bestandteil zur Behandlung des plötzlichen Herztodes (PHT) bei Patienten mit entsprechendem Risikoprofil. Während im Rahmen einer Sekundärprophylaxe die ICD-Versorgung nach Ausschluss passagerer Ursachen bzw. bestehender Kontraindikationen zeitnah erfolgen sollte, empfehlen die aktuel- 


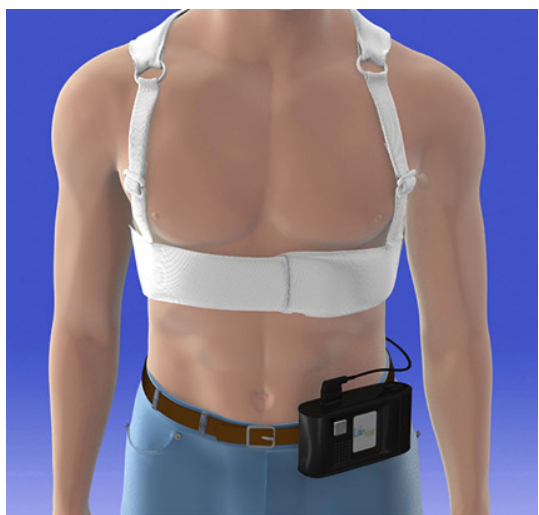

Abb. 1 ॥ Wearable cardioverter-defibrillator (LifeVest ${ }^{\circledR}$, Zoll CMS GmbH, Köln, Deutschland). (Mit freundl. Genehmigung durch Zoll CMS, Deutschland)

len Guidelines der European Society of Cardiology (ESC) [2] sowie auch die Deutsche Gesellschaft für Kardiologie - Herz- und Kreislaufforschung (DGK) im deutschen Kommentar dieser Leitlinien [1] ein zunächst zurückhaltendes und abwartendes Handeln im Falle einer Primärprävention des PHT. Diese Empfehlungen basieren auf den Erkenntnissen, dass es bei einem substanziellen Patientenanteil unter einer adäquaten medikamentösen Therapie zur Verbesserung der kardialen Pumpfunktion kommt [3]. Daher sollte gerade Patienten nach einem akuten Myokardinfarkt, einer Koronarintervention (PCI) bzw. operativen Myokardrevaskularisation (CABG) und/oder einem Herzklappeneingriff eine angemessene myokardiale Erholungszeit eingeräumt werden. Hierbei ist jedoch zu bedenken, dass während dieser Krankheitsphase ein erhöhtes Risiko [4] zum Auftreten lebensbedrohlicher Arrhythmien besteht, welches sich weder durch eine angemessene Herzinsuffizienzmedikation noch durch die additive Gabe von Antiarrhythmika entscheidend beeinflussen lässt. So kann es bis zu drei Monate andauern, ehe es $\mathrm{zu}$ einer relevanten kardialen Erholung mit Reduktion des PHT-Risikos kommt und eine abschließende, sichere und stete ICD-Indikation gestellt werden kann. So untersuchte die DINAMIT-Studie (The Defibrillator in Acute Myocardial Infarction Trial, [5]) an 674 Patienten mit einer Follow-upPeriode von 30 Monaten, ob sich die
Mortalität durch die primärprophylaktische ICD-Implantation in der Akutbzw. Frühphase nach Myokardinfarkt bzw. Koronarrevaskularisation im Vergleich zur konservativen Therapie ohne Defibrillatorimplantation beeinflussen lässt. Hierbei fand sich jedoch mit $62 \mathrm{zu}$ 58 Todesfällen kein signifikanter Mortalitätsunterschied. Allerdings ergab die detailliertere Datenanalyse, dass sich die Anzahl der arrhythmiebedingten Todesfälle in der ICD-Gruppe mit 12 Fällen im Vergleich zu 29 sehr wohl unterschied, ebenso wie umgekehrt die Anzahl der nichtarrhythmieassoziierten Todesfälle mit 50 in der ICD-Gruppe zu nur 29 in der Kontrollgruppe. Kongruente Aussagen ergaben sich im IRIS-Trial (Immediate Risk Stratification Improves Survival trial, [6]). In einem analogen Studiensetting wurden 898 Patienten mit einem Follow-up von 37 Monaten untersucht. Hierbei zeigten sich ebenfalls deutlich weniger Fälle des PHT mit $27 \mathrm{zu}$ 60 in der ICD-Gruppe im Vergleich zur ICD-freien Kontrollgruppe bei einer vergleichbaren „Overall“-Mortalität von 116 zu 117. Folglich scheint die umfängliche Rhythmusdetektion mit der Möglichkeit zur automatisierten Defibrillation durchaus die arrhythmiebedingte Mortalität während der hier diskutierten Frührisikophase zu beeinflussen. Erklärungen für die ähnliche Letalität mit und ohne ICD könnten sich, neben individuellen lebensbedrohenden Komorbiditäten, in der zusätzlichen Belastung des Eingriffs sowie in eingetretenen Komplikationen durch das Implantat selbst wiederfinden. Auch könnte das implantierte Aggregat nach ventrikulärer Rekonvaleszenz mit Wiederanstieg der Pumpfunktion und der darin begründeten Reduktion des Arrhythmierisikos gar nicht mehr indiziert sein. Diese Tatsache hätte in Bezug auf das implantierte ICD-System die Konsequenz, dass ein Patient bei fehlendem Benefit ausschließlich das Risiko von ICD-assoziierten Komplikationen zu tragen hätte, wie beispielweise inadäquate Defibrillatortherapien (18-22\%), Systeminfektionen (6\%) oder Sondenkomplikationen (17\%) [7].

Diese Erkenntnisse mündeten in der aktuellen Leitlinienempfehlung, eine primärprophylaktische ICD-Implantation nicht innerhalb der ersten 40 Tage nach einem Myokardinfarkt durchzuführen und die Entscheidung für oder gegen eine permanente ICD-Versorgung erst nach Reevaluation der linksventrikulären Ejektionsfraktion (LV-EF) 6 bis 12 Wochen nach dem akuten Ischämieereignis $\mathrm{zu}$ treffen $[1,2]$. Analoge Empfehlungen gelten auch für die koronare Bypassoperation (CABG) und die perkutane Koronarintervention (PCI). Weiterhin werden nach Erstdiagnose einer nichtischämischen Kardiomyopathie eine leitliniengerechte Herzinsuffizienztherapie für mindestens 3 Monate und eine anschließende Reevaluation von LV-EF und New York Heart Association(NYHA)-Klasse empfohlen, bevor eine implantatbasierte Therapieeskalation erfolgen sollte $[1,2]$.

Einen Ausweg aus dem Dilemma eines einerseits fehlenden Defibrillatorschutzes in der Frühphase des ventrikulären Remodeling und der andererseits $\mathrm{zu}$ frühzeitigen Implantation eines möglicherweise nicht dauerhaft indizierten ICD-Systems stellt der Einsatz eines tragbaren („wearable“) KardioverterDefibrillators (WCD) dar. Dieser bietet während dieser rhythmussensiblen „Bridging“- und Evaluationsphase eine sichere Therapieoption in der Behandlung des PHT. Während sich jedoch der WCD-Einsatz in kardiologischen Fachabteilungen bereits zunehmend etabliert hat, findet diese Therapieoption in herzchirurgischen Fachabteilungen bisher eine eher zurückhaltende Anwendung. Daher ist es Ziel dieses Positionspapiers, die Indikationen für den Einsatz des WCD vor- und nach kardiochirurgischen Eingriffen aufzuzeigen und Herzrhythmus- bzw. Herzinsuffizienzteams eine praxisnahe Entscheidungshilfe zu geben.

\section{Aufbau und Funktionsweise des tragbaren Kardioverter- Defibrillators}

Das einzige, aktuell auf dem Markt verfügbare Modell eines WCDs (LifeVest ${ }^{\circledR}$, Zoll CMS GmbH, Köln, Deutschland) besteht aus einem tragbaren Westenteil, das vier Detektions- und drei Defibrillationselektroden beinhaltet und einer bat- 
Z Herz- Thorax- Gefäßchir 2018 · 32:286-299 https://doi.org/10.1007/s00398-018-0246-6

(c) Der/die Autor(en) 2018

\section{H. Burger · J. Schmitt · M. Knaut · T. Eitz · C. T. Starck · S. Hakmi · A. Siebel · A. Böning}

\section{Einsatz des tragbaren Kardioverter-Defibrillators nach kardiochirurgischen Eingriffen. Positionspapier der AG Herzrhythmusstörungen der Deutschen Gesellschaft für Thorax-, Herz- und Gefäßchirurgie}

\section{Zusammenfassung}

Der implantierbare Kardioverter-Defibrillator (ICD) stellt seit Jahrzehnten den Goldstandard in der Primär- und Sekundärprophylaxe des plötzlichen Herztodes (PHT) für Patienten mit definiertem Hochrisikoprofil dar. Allerdings mahnen die aktuellen Leitlinien zur Zurückhaltung bei der frühzeitigen primärpräventiven ICD-Implantation im Rahmen erstmalig erhobener Risikokonstellationen. Auf diese Weise soll die Implantation eines, im weiteren Krankheitsverlauf nicht mehr indizierten ICDs vermieden werden. Anstelle dessen ist diesen Patienten eine angemessene Zeit zur Restitution der kardialen Grunderkrankung unter leitliniengerechter Therapie zu gewähren. Indikationsbereiche, bei denen Zurückhaltung empfohlen wird, stellen u. a. herzchirurgische Eingriffe wie koronare Revaskularisationsoder Herzklappeneingriffe dar, insbesondere bei Patienten mit einer perioperativen linksventrikulären Ejektionsfraktion (LV-EF) von $35 \%$ oder geringer. Oftmals kommt es hierbei nämlich nach einer RemodelingPhase zur deutlichen Erholung der kardialen Pumpfunktion mit Wiederanstieg der LVEF. Folglich entfällt zu diesem Zeitpunkt die leitliniengerechte ICD-Indikation. Andererseits besteht gerade für diese Patienten während der Restitutionsphase ein hohes Risiko zum Auftreten von lebensbedrohlichen Herzrhythmusstörungen und somit für den PHT.

Vor diesem Hintergrund kommt dem tragbaren Kardioverter-Defibrillator (WCD) eine besondere Bedeutung zu. Dieser kann als passageres Defibrillatorsystem Patienten zuverlässig und effektiv vor dem PHT schützen. Aus diesem Grund ist der Einsatz eines WCD nach aktuellen Leitlinien indiziert, wenn zum einen eine passagere, möglicherweise reversible Phase von erhöhtem PHT-Risiko vorliegt oder zum anderen die Extraktion eines infizierten ICD-Systems mit der temporären Kontraindikation zur zeitgleichen Neuimplantation eines ICD-Systems besteht. Nachfolgend werden daher neben der WCDFunktionsweise die Anwendungsgebiete in der Kardiochirurgie, die aktuellen Studienund Leitlinienempfehlungen sowie die Implementierung eines WCD-Programms in den klinischen Alltag aufgezeigt, um Ärzten eine praxisgerechte Hilfestellung für den klinischen Einsatz zu geben.

Schlüsselwörter

Tragbarer Defibrillator · Implantierbarer Kardioverter-Defibrillator · Sondenextraktion . Positionspapier - Arbeitsgemeinschaft Herzrhythmusstörungen

\section{Use of wearable cardioverter defibrillators after cardiac surgery. Position paper of the working group on cardiac arrhythmias of the German Society for Thoracic and Cardiovascular Surgery}

\begin{abstract}
Implantable cardioverter defibrillators (ICD) have been the gold standard for primary and secondary prophylaxis of sudden cardiac death (SCD) in defined high-risk patients for decades; however, recent guidelines sound a note of caution with the early ICD implantation in patients with newly found risk constellations to avoid implantation of an ICD that would not be indicated during later stages of the disease. Instead of early implantation, these patients should be given a reasonable amount of time for cardiac recovery on medicinal treatment according to the guidelines. The ICD should be used with caution after cardiac surgery, such as revascularization or valvular interventions,
\end{abstract}

especially in patients with a perioperative left ventricular ejection fraction (LV-EF) of $35 \%$ or lower. Left ventricular function often makes a considerable recovery with a rise in LV-EF after cardiac remodelling. Therefore, a valid indication for ICD implantation no longer exists; however, especially for patients in the recovery period, a high risk for life-threatening rhythm disturbances and SCD still remains. Against this background wearable cardioverter defibrillators (WCD) become more important because they can protect patients safely and effectively from SCD. Therefore, the use of a WCD is indicated according to the current guidelines if a transient, possibly reversible period of an increased SCD risk exists or if after explantation of an infected ICD there is a contraindication for a simultaneous re-implantation of the device. This manuscript, therefore shows the technical aspects of the WCD, indications for patients after cardiac surgery, current recommendations from studies and guidelines as well as the implementation of a WCD program in the daily routine to give medical professionals practice-oriented assistance for the clinical implementation.

\section{Keywords}

Wearable defibrillator - Implantable cardioverter defibrillator - Lead extraction - Position paper - Working group Cardiac Arrhythmia terietragenden Steuereinheit (• Abb. 1). Durch diese Anordnung werden die kontinuierliche Rhythmusanalyse durch zwei EKG-Ableitungen und eine etwaige Defibrillation ermöglicht. Weiterhin erhält der Patient eine entsprechende Akkuladestation, die zusätzlich eine Funkverbindung zur Steuereinheit der Weste aufbauen kann und auf diese Weise das Bindeglied zur Datenübertra- gung an eine internetbasierte Datenbank (LifeVest-Network $^{\circledR}$ ) darstellt. Diese Verknüpfung ist sinnvoll, da die LifeVest ${ }^{\circledR}$ zusätzlich auffällige EKG-Sequenzen aufzeichnen kann, vergleichbar eines Event-Rekorders. Die so gewonnenen Informationen über Arrhythmie-, Bradykardie- oder Asystoliephasen werden anschließend übertragen und können umgehend durch den behandelnden
Arzt über einen passwortgeschützten Zugang eingesehen und analysiert werden. Vor der Systemübergabe erhält jeder Patient zwingend eine ausführliche Schulung, um den richtigen Umgang mit dem WCD sicherzustellen.

Das aktive LifeVest-System überwacht und therapiert den Patienten während der gesamten Tragezeit. Daher sollte dieses möglichst $24 \mathrm{~h}$ getragen und nur 


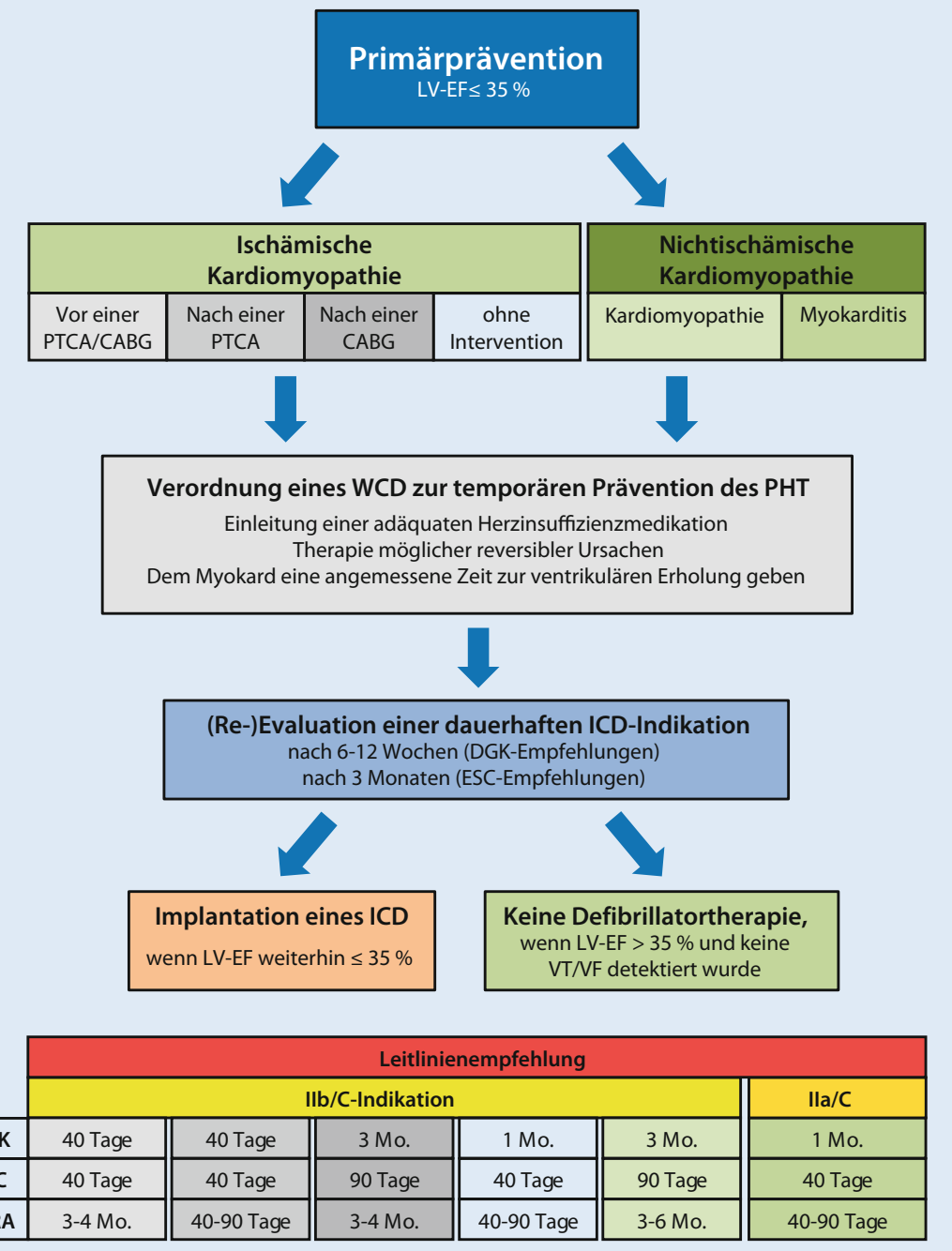

Abb. 2 ム Übersicht der primärpräventiven WCD-Indikationen. PTCA perkutane transluminale Koronarangioplastie, $C A B G$ "coronary artery bypass grafting ", WCD "wearable cardioverter-defibrillator", $I C D$ „implantable cardioverter-defibrillator", DGK Deutsche Gesellschaft für Kardiologie, ESC European Society of Cardiology, EHRA European Heart Rhythm Association, Mo. Monate, LV-EF linksventrikuläre Ejektionsfraktion, PHT plötzlicher Herztod, VT ventrikuläre Tachykardie, VF ventricular fibrillation

unter Überwachung einer weiteren Person, beispielsweise zur Körperhygiene, abgelegt werden. Erkennt das WCDSystem eine potenziell lebensbedrohliche ventrikuläre Herzrhythmusstörung, warnt es den Patienten durch die Abgabe eines lauten Signaltons, eines hellen Lichtkegels sowie durch einen kräftigen Vibrationsalarm. Zu diesem Zeitpunkt hat der Patient die Gelegenheit, eine möglicherweise ungerechtfertigte Schockabgabe durch Drücken zweier Reaktionstasten zu unterbinden. Unterbleibt eine solche Reaktion, geht das Gerät von einem bewusstlosen, behandlungsbedürftigen Patienten aus und stößt elektrischen Widerstand und schützt die Haut vor Verbrennungen während des unmittelbar folgenden 150-J-Schocks. Parallel hierzu werden umstehende Personen mittels Sprachinformationen auf die Notfallsituation hingewiesen und aufgefordert, den Patienten während der Defibrillation nicht zu berühren sowie einen Notruf abzugeben. Insgesamt kann das Gerät bis zu fünf Schockabgaben pro Sequenz nach jeweiliger Redetektion abgeben. Die Rhythmusdetektion erfor- dert $10-15 \mathrm{~s}$, und bis zur Schockabgabe vergehen $30-40 \mathrm{~s}$.

Zishiri et al. [13] konnten bei 4958 Patienten nach einer aortokoronaren Bypass-Operation mit hochgradig reduzierter LV-EF aufzeigen, dass es bei 809 Patienten des WCD-versorgten Kollektivs in 1,3\% der Fälle zu gerechtfertigten Schockabgaben (18 Schocks bei 11 Patienten) kam. Weiterhin ergaben Analysen einen Überlebensvorteil für WCD-Träger (Mortalität 2\%) im Vergleich zu Patienten ohne WCD (7\% Mortalität) innerhalb der ersten 90 Therapietage. Dieser Trend [6] bestätigte sich zudem in der anschließenden 3-jährigen Beobachtungsphase. Allerdings wurden auch 13 unangemessene Schockabgaben dokumentiert. In einem weniger selektionierten Patientenklientel erfassten Wäßnig et al. [8] hingegen deutlich weniger inadäquate Schockabgaben mit 26 von 6043 (entsprechend $0,4 \%$ ) und 163 gerechtfertigten Schockabgaben $(1,6 \%)$ bei 120 Patienten mit einer effektiven Rhythmuskonversion in $94 \%$ der Fälle.

Im Vergleich zu einem permanent implantierten Defibrillator kann der WCD allerdings keine antibradykarde oder antitachykarde Stimulation (ATP) durchführen, auch nicht als „post-shock pacing" bei Asystolie nach erfolgter Kardioversion bzw. Defibrillation. Diese Einschränkungen spielen jedoch laut Wäßnig et al.im Rahmen der hier betrachteten Krankheitsbilder eine eher untergeordnete Rolle [8]. Insgesamt zeichnet sich der WCD durch eine hohe Funktionalität und Wirksamkeit aus, und es ergeben sich Erfolgsraten von bis zu $98 \%$ [9].

\section{Bisherige klinische Erfahrungen mit dem WCD}

Seit der Zulassung des WCDs durch die FDA, der CE-Zertifizierung und der klinischen Einführung im Jahre 2001 konnten zahlreiche pro- und retrospektive Register sowie mehrere Studien die Sicherheit und Effektivität des WCD in der Prävention des PHT mit mehr als 10.000 eingeschlossen Patienten belegen [8-14]. Weiterhin wurden unlängst die Ergebnisse eines retrospektiven WCDRegisters [8] von über 6000 behandelten Patienten aus über 400 deutschen Klini- 
ken publiziert. Wäßnig et al. [8] zeigten hier in einem heterogenen Patientenkollektiv, dass nicht nur die Compliance mit einer täglichen Tragezeit von durchschnittlich 23,1 h sehr hoch war, sondern auch, dass 1,6\% der Patienten aufgrund maligner ventrikulärer Arrhythmien (VT/VF) durch eine Defibrillation adäquat behandelt werden konnten. Dies entspricht etwa 8,4 Ereignissen/100 Patientenjahre mit einer Überlebensrate von über $93 \%$ binnen der ersten $24 \mathrm{~h}$ nach Therapieabgabe. Zudem wurde eine hohe Anzahl an selbstlimitierenden VT-Ereignissen erfasst, bei denen eine Schockabgabe vom Patienten durch das zeitgemäße Betätigen der Reaktionstasten unterbunden wurde. Kutyifa et al. konnten in einem prospektiven Register (Wearable Defibrillator in Terminating Tachyarrhythmias Trial, WEARIT-II; [10]) zeigen, dass sich $41 \%$ der Patienten in der LV-EF wieder besserten und so nach Abschluss der WCD-Versorgung nur noch $42 \%$ einen permanenten ICD benötigten. Weiterhin zeigten Duncker et al. (Prolongation of Reverse remOdelling period to avoid untimely ICD impLantation in newly diagnOsed heart failure usiNG the wearable cardioverter/ defibrillator-PROLONG-Study) [15], dass es bei nichtischämischen Patienten auch 4 bis 6 Monate nach Erstdiagnose noch zur Verbesserung der LV-EF kommen kann. Zudem beobachteten sie, dass es in rund $7 \%$ der Fälle während dieser Evaluationsphase zur adäquaten WCD-Therapieabgabe kam [16]. Daher forderten die Autoren, dass auch diese Patienten zunächst mit einem WCD versorgt werden sollten. Die aktuellsten Studienergebnisse stellte im März dieses Jahres der Kostudienleiter Jeffrey Olgin über die noch nicht publizierte prospektive randomisierte, kontrollierte VESTStudie (Vest Prevention of Early Sudden Death Trial) auf der 67. Jahrestagung des American College of Cardiology (ACC) in Orlando, USA, vor. Dieses multizentrische Register schloss 2302 Patienten mit einer koronaren Herzerkrankung nach einem akuten Myokardinfarkt mit einer LV-EF $\leq 35 \%$ sowie erfolgter oder nichtdurchgeführter Koronarrevaskularisation (PCI bzw. CABG) ein und beinhaltete eine 90-tägige Follow-up-
Periode. Untersucht wurde, ob sich zwischen einer alleinigen leitliniengerechten medikamentösen Therapie (778 Patienten) und einer additiven WCD-Versorgung (1524 Patienten) Unterschiede in der Häufigkeit des PHT und der Gesamtmortalität ergeben. Erste Ergebnisse zeigen nun, dass sich durch die additive WCD-Therapie zwar ein Trend, aber keine statistisch signifikante Reduktion der arrhythmieassoziierten Mortalität mit $1,6 \% \mathrm{zu} 2,4 \%(p=0,18)$, der nichtrhythmogenbedingten Todesfälle mit $1,4 \% \mathrm{zu}$ $2,2 \%(p=0,15)$ sowie der schlaganfallbedingten Letalität $0 \% \mathrm{zu} 0,5 \%(p=0,01)$ nachweisen ließen. Insbesondere reduzierte sich die Gesamtmortalität in der WCD-Gruppe deutlicher auf 3,1\% im Vergleich zur medikamentösen Gruppe mit $4,9 \%(p=0,04)$, was einer relativen Reduktion von $36 \%$ entspricht. Betrachtet man die bisherigen WCD-Analysedaten, so ergeben sich $1,4 \%$ adäquate und $0,6 \%$ inadäquate Schockabgaben und eine WCD-Tragezeit von 14,1 h/Tag [53].

Letztlich führten die vorliegenden $\mathrm{Da}$ ten bereits im Jahre 2015 zur Aufnahme des WCDs in die europäischen Leitlinien [2] und 2017 in den nationalen Kommentar der europäischen Leitlinien zur Behandlung ventrikulärer Arrhythmien [1].

\section{Indikationen zum Einsatz des WCD im Rahmen kardiochirurgischer Eingriffe}

Anhand internationaler Leitlinien und nationaler Kommentare zur Behandlung ventrikulärer Arrhythmien ergeben sich aktuell für die WCD-Anwendung Indikationen mit einem Evidenzlevel von I/C-IIb/C [1, 2, 17-19]. Die darin benannten Krankheitsbilder schließen auch kardiochirurgische Patienten ein, die ein temporär erhöhtes PHT-Risiko oder eine vorübergehende Kontraindikation zur ICD-Implantation bei bestehender ICD-Indikation aufweisen. Insbesondere postoperative Patienten mit einer hochgradig reduzierten LV-EF zeigen über einen längeren Zeitraum ein erhöhtes Risiko für ventrikuläre Arrhythmien [20], weshalb sich eigentlich eine ICDImplantation begründen ließe. Aller- dings belegen mehrere Untersuchungen, dass es nach einer angemessenen Zeit in zahlreichen Fällen zur Restitution der Ventrikelfunktion kommen kann und daher eine $\mathrm{zu}$ frühzeitige ICDVersorgung keine dauerhafte ICD-Indikation beinhalten würde [7, 21, 22]. Daher wird im Folgenden detaillierter dargestellt, welche Patienten nach herzchirurgischen Eingriffen für eine vorübergehende WCD-Therapie geeignet erscheinen.

\subsection{Primärprophylaktische Indikationen}

\subsubsection{Koronare Herzerkrankung, ischämische Kardiomyopathie, Myokardinfarkt und Myokardre- vaskularisation}

Die Auswirkungen einer koronaren Herzerkrankung werden Patienten oftmals erst zum Zeitpunkt des Auftretens von hochgradigen Koronarstenosen oder akuten Gefäßverschlüssen durch die Symptome eines Angina-pectoris-Anfalls oder Myokardinfarkts offensichtlich. So gelangen etwa $10-20 \%$ der kardiochirurgischen Patienten aufgrund eines akuten Myokardinfarkts zur dringenden operativen Myokardrevaskularisation. Auch wenn sich unter diesen Patienten nur ein geringer Anteil mit einer hochgradig eingeschränkten LV-EF ( $\leq 35 \%)$ befindet, besteht doch insbesondere für diese ein erhöhtes Risiko zum Auftreten maligner peri- und postoperativer Arrhythmien. Denn einerseits kann es in den ersten Stunden nach erfolgter Reperfusion der Infarktareale zum Effekt des „reperfusion injury“ durch pH-Wertund Elektrolytverschiebungen kommen [24, 25], und andererseits können sich lebensbedrohliche Arrhythmien während der anschließenden ventrikulären Remodeling-Phase durch das absterbende Myokard der irreversibel geschädigten Infarktareale entwickeln [24-29]. Aber auch inkomplette Revaskularisationen bedingen im weiteren Krankheitsverlauf eine grundsätzlich erhöhte Mortalität im Vergleich zu kompletter Revaskularisation, so eine aktuelle Studienübersicht von Zimarino et al. [51]. Speziell bei Patienten mit einer hochgradig eingeschränkten LV-EF und ICD-Indikation 


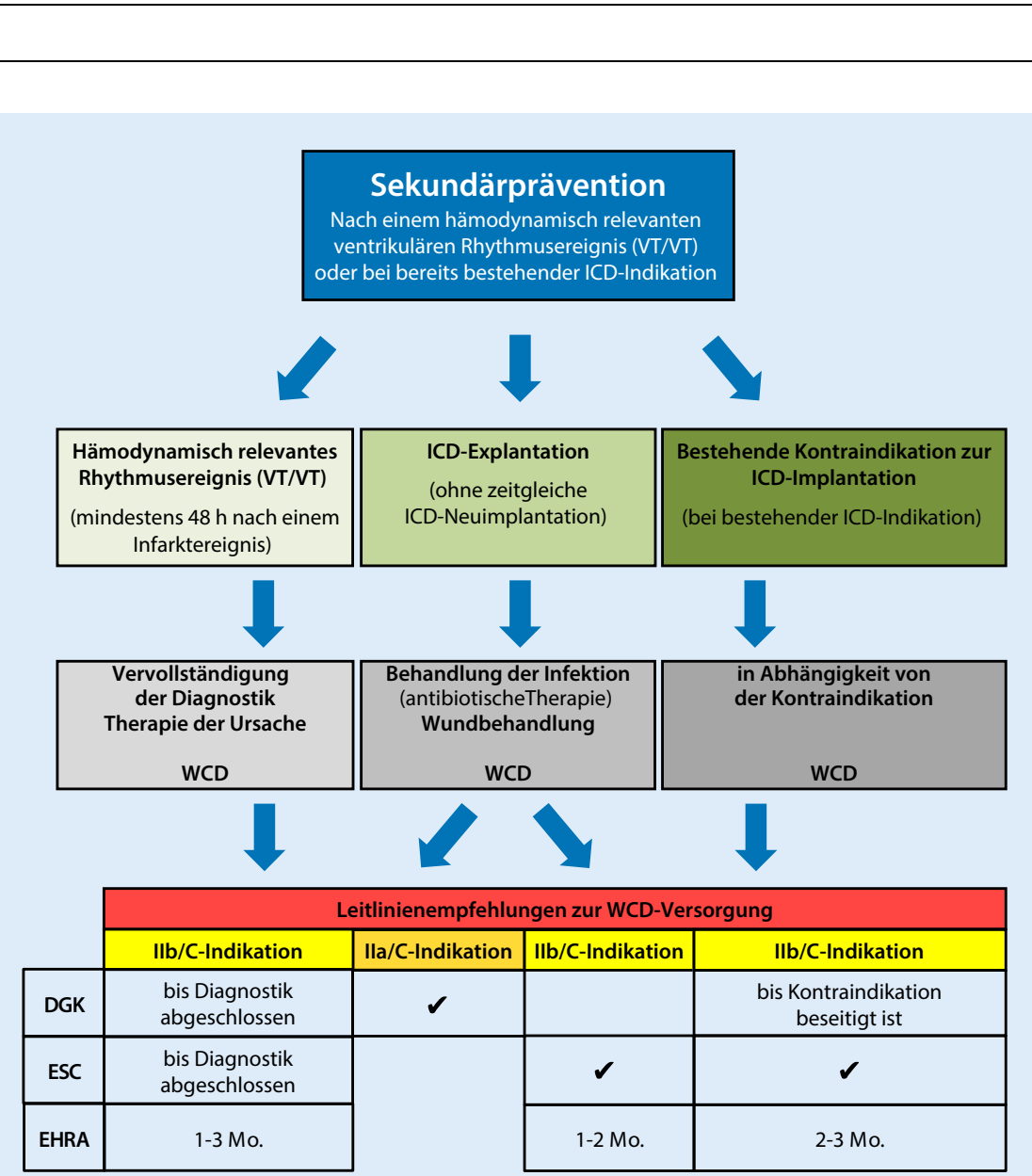

Abb. 3 A Übersicht der sekundärpräventiven WCD-Indikationen. VF ventricular fibrillation, VT ventrikuläre Tachykardie, $W C D$,wearable cardioverter-defibrillator", ICD ,implantable cardioverter-defibrillator", DGK Deutsche Gesellschaft für Kardiologie, ESC European Society of Cardiology, EHRA European Heart Rhythm Association, $\checkmark$ Empfehlung ohne genaue Zeitangaben, Mo Monate

weisen Untersuchungen von NombelaFranco et al. [52] die inkomplette Revaskularisation als unabhängigen Prädiktor zum Auftreten ventrikulärer Arrhythmien nach. Weiterhin belegten Zishiri et al. [13], dass Patienten mit einer LV$\mathrm{EF} \leq 35 \%$ innerhalb der ersten 90 Tage nach erfolgter koronarer Bypass-Operation bzw. PCI einem erhöhten PHTRisiko unterliegen: Die Kaplan-MeierÜberlebenskurven dieser Untersuchung zeigten einen Überlebensvorteil innerhalb dieser Periode für Träger eines WCDs. Hier ergaben sich nach erfolgter Bypass-Operation Mortalitäten von 3\% mit WCD gegenüber $7 \%$ ohne diesen zusätzlichen Schutz und nach PCI von $2 \% \mathrm{zu} 10 \%$. Allerdings gelang es den Autoren nicht, einen statistisch signifikanten rhythmogenen Zusammenhang zu dieser Mortalitätsdifferenz nachzuweisen. Weiterhin zeigte die STICH- im weiteren Verlauf einen dauerhaften ICD. Eine WCD-Verordnung scheint so insbesondere während der postoperativen Periode - vergleichbar der Postinfarktphase - für Patienten mit einer LV-EF $\leq 35 \%$ für bis zu 3 Monaten empfehlenswert, wenn eine adäquate, stationäre Rhythmusüberwachung, wie beispielweise aufgrund einer Patientenverlegung zur Anschlussheilbehandlung, nicht mehr gewährleistet werden kann.

Aber auch eine schleichende chronische Minderdurchblutung der Herzmuskulatur kann im Vergleich zu den oben beschriebenen, akuten Krankheitsverläufen ernste Konsequenzen bedingen und in eine vorübergehende oder dauerhafte Herzinsuffizienz auf Basis einer ischämischen Kardiomyopathie münden. Hierbei ist die weitere Krankheitsprognose besonders von den bisher eingetretenen ischämischen Myokardschäden wie der Größe reversibler ventrikulärer "Stunning“-Areale oder bereits bestehender irreversibler Infarktnarben abhängig. Kommt es in deren Folge zum myokardialen Pumpkraftverlust mit einer LV-EF-Reduktion $\leq 35 \%$, einer Herzinsuffizienz (NYHA-Stadium $\geq$ II) und einer konsekutiven Ventrikeldilatation als Ausdruck der eingetretenen ischämischen Kardiomyopathie, so ergibt sich anhand der aktuellen nationalen und internationalen Leitlinien $[1,2]$ und den Erkenntnissen der MADIT-II-Studie [23] die Indikation zur dauerhaften primärprophylaktischen ICD-Implantation. Jedoch ist dieser Zustand nicht immer irreversibel und dank spezieller Untersuchungsmethoden wie einer Myokardszintigraphie, Stressechokardiographie oder eines Kardio-MRTs ist es heutzutage häufig möglich, in diesen geschädigten Arealen passives jedoch noch vitales Myokardgewebe von irreversibel vernarbtem Gewebe $\mathrm{zu}$ unterscheiden. Gelingt es auf diese Weise, ausreichende Areale von "hibernating myocardium" zu detektieren, so ergibt sich ein sinnvoller Ansatz für eine koronare Revaskularisation mit einer günstigen Prognose zur Restitution der kardialen Pumpfunktion. Daher kann für diese Patienten für bis zu 40 Tage vor dem geplanten Eingriff ein vorüberge- 


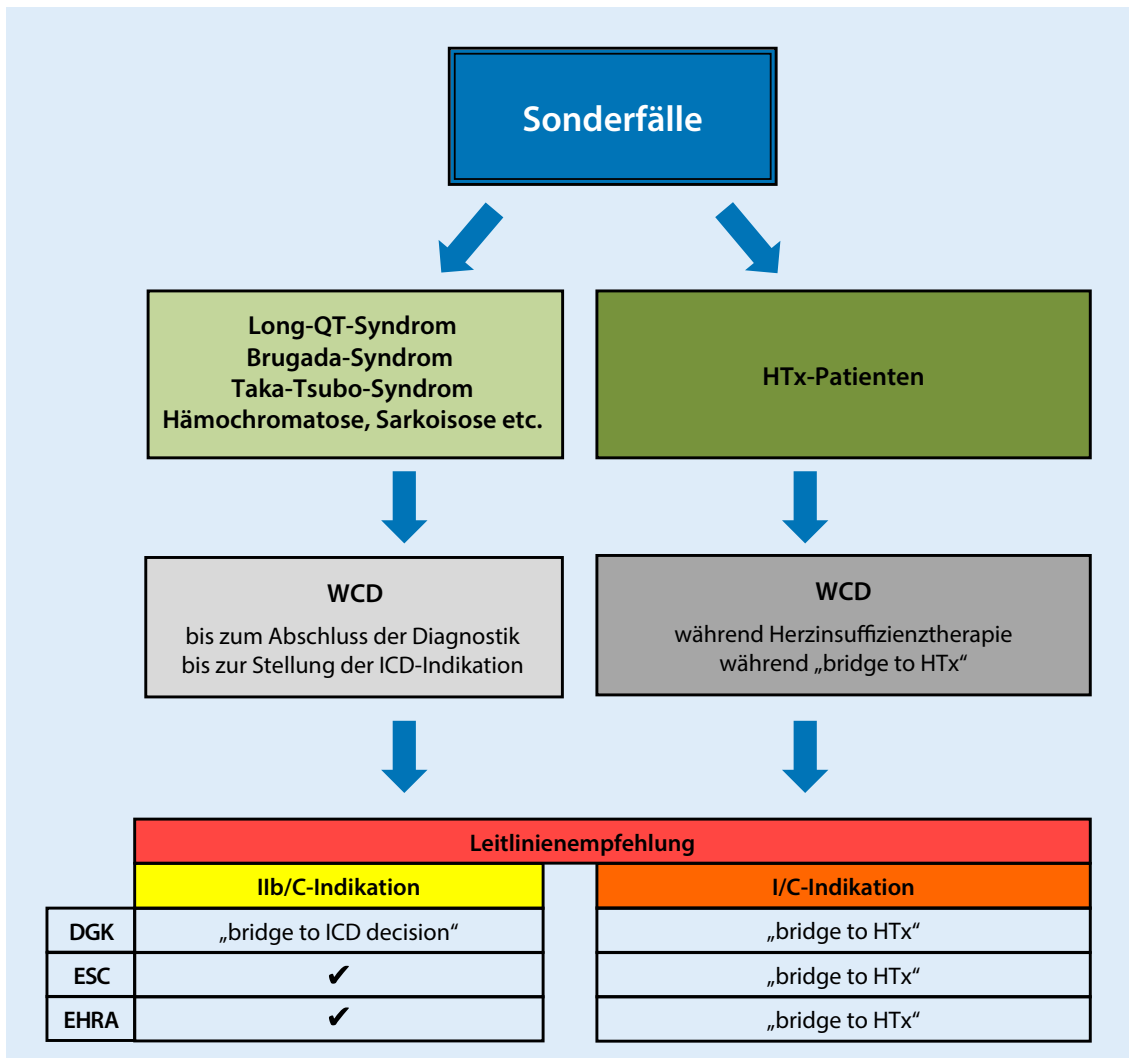

Abb. 4 ム Übersicht der Sonderfälle der WCD-Indikation. HTx Herztransplantation, WCD "wearable cardioverter-defibrillator", ICD "implantable cardioverter-defibrillator", $D G K$ Deutsche Gesellschaft für Kardiologie, ESC European Society of Cardiology, EHRA European Heart Rhythm Association, $\checkmark$ Empfehlung ohne genaue Zeitangaben

hender WCD zur Prophylaxe des PHT verordnet werden $[1,2,10]$.

Zusammenfassend ist nach aktuellem Wissensstand der Einsatz eines WCDsfür Patienten mit einer fortgeschrittenen koronaren Herzerkrankung und einer LV$\mathrm{EF} \leq 35 \%$ zu empfehlen,

1. während diese auf eine Herzoperation warten und

2. während der Rekompensationsperiode nach einem akuten Myokardinfarkt für bis zu 3 Monate bzw. 3 bis 4 Monate nach erfolgter koronarer Revaskularisation (Klasse-IIb/CEmpfehlung) [1, 2, 18, 31, 32].

3. und die Therapie sollte nach angemessener postoperativer Restitution und echokardiographischen Kontrollen zur Reevaluation der Pumpfunktion mit der Entscheidung über die dauerhafte ICD-Indikation unter Berücksichtigung der klinischen Herzinsuffizienzsymptomatik (NYHA) enden (• Abb. 2).
Aussagen zur Inzidenz dieser Rhythmusstörungen in der gegenwärtigen Literatur $[9,33,34]$. So empfehlen die aktuellen Leitlinien nach Erstdiagnose einer DCM mit hochgradig reduzierter LV-EF ( $\leq 35 \%)$ das umgehende Einleiten einer optimierten Herzinsuffizienzmedikation, die Verordnung eines WCD (Klasse-IIb/C-Empfehlung) und die anschließende Befundreevaluation mit der Entscheidung über eine dauerhafte ICDIndikation nach 3 Monaten $[1,2,35]$. Dieses abwartende Vorgehen ist nach Kao et al. [36] insbesondere deswegen gerechtfertigt, da es bei bis zu $41,5 \%$ dieser Patienten unter einer adäquaten Medikation zum Wiederanstieg der LV$\mathrm{EF}$ auf über $40 \%$ kommen kann und sich somit keine ICD-Indikation mehr ergibt. Zudem ermöglicht der WCD während dieser Akutphase die Dokumentation von unklaren Rhythmusereignissen, wodurch sich zusätzliche diagnostische Hinweise zur weiteren Risikostratifikation ergeben können.

Weiterhin kann der WCD überbrückend bis zur abschließenden individuellen Indikationsstellung bei Verdacht auf andere, nichtischämisch bedingte Kardiomyopathien mit hochgradig reduzierter LV-EF verordnet werden, wie beispielsweise der peri- oder postpartalen Kardiomyopathie, dem BrugadaSyndrom oder einer Tako-Tsubo-Kardiomyopathie (Klasse-IIb/C-Indikation [1, 2], - Abb. 4). Insbesondere für den Fall einer peripartalen Kardiomyopathie empfehlen Duncker et al. [37] eine 3- bis 6-monatige WCD-Periode zur Protektion vor dem PHT, da sie hierbei bis $\mathrm{zu} 12 \%$ an behandlungsbedürftigen ventrikulären Arrhythmien in einem Beobachtungszeitraum von 4 Monaten nachweisen konnten.

Letztlich profitieren auch Patienten mit akuten Myokarditiden und reduzierter LV-EF von einer WCD-Versorgung (Klasse-IIa/C-Empfehlung [1, 2]). Im Rahmen dieses Krankheitsbildes ist von etwa $11 \%$ an relevanten ventrikulären Arrhythmien binnen der ersten 11 Monate auszugehen, wie es Konrad et al. [38] berichten. Bemerkenswerterweise beziehen sich diese Daten auf ein Patientenkollektiv, welches eine nur leicht bis mittelgradig reduzierte LV-EF (43-50\%) 


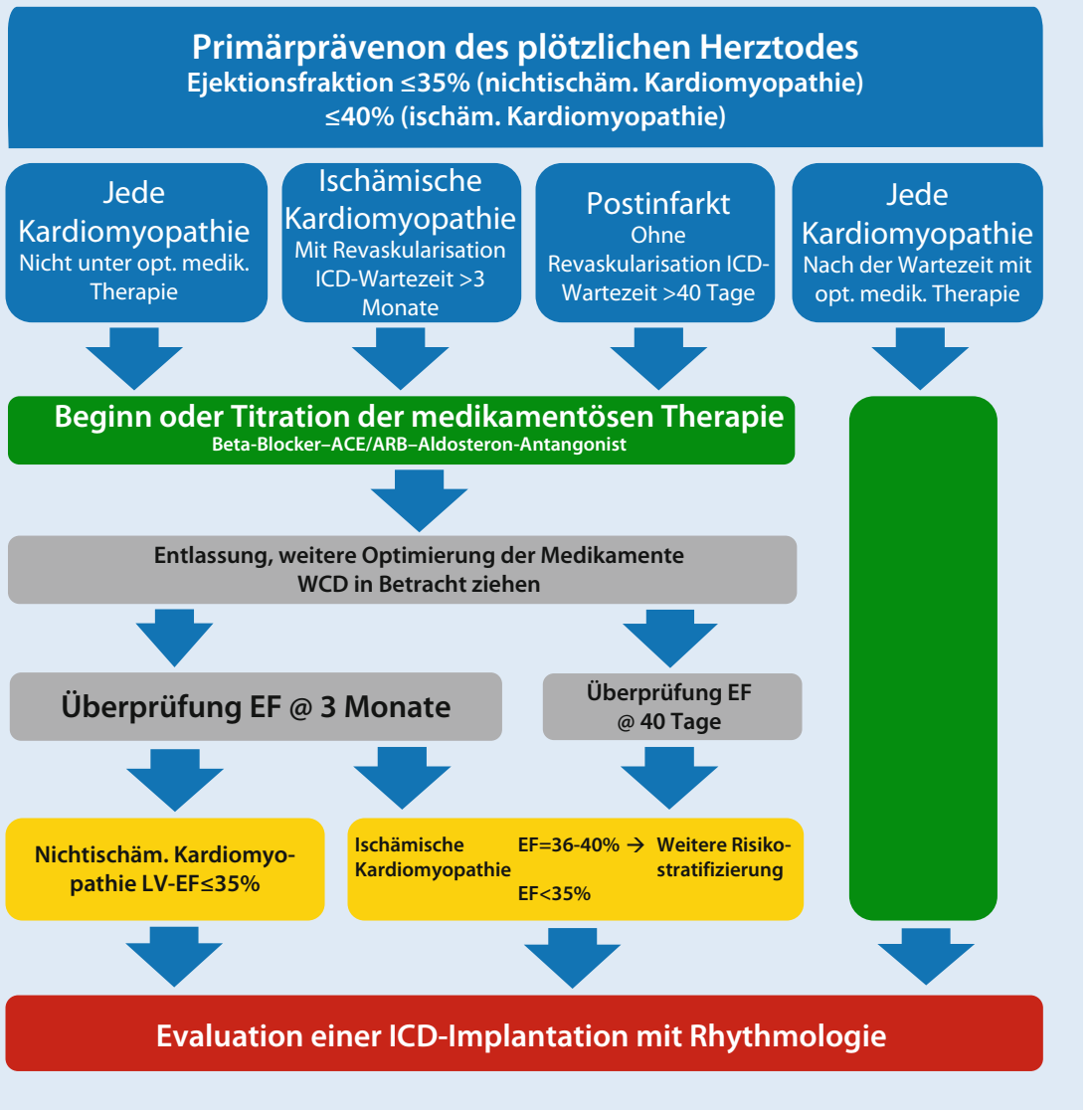

Abb. 5 ॥ Schaubild - klinische Behandlungspfade für herzinsuffiziente Patienten. EF/LV-EF linksventrikuläre Ejektionsfraktion, ICD Implantable Cardioverter-Defibrillator, WCD Wearable CardioverterDefibrillator, ischäm. ischämische, nichtischäm. nichtischämische, opt. medik. optimierte medikamentöse, @ für, $A C E / A R B$ angiotensin converting enzyme/angiotensin-receptor blocker. (In Anlehnung an www.HRSonline.org)

aufwies, und so ist sogar eine noch höhere Inzidenz bei Patienten mit höhergradig reduzierter LV-EF ( $\leq 35 \%)$ zu befürchten. Kindermann et al. [39] schilderten zudem, dass sich das höchste Risiko für den PHT innerhalb der ersten 18 Monate nach akuter Myokarditis ergibt. Somit sollte in jedem Fall die Diagnose einer Myokarditis auf Echokardiographie-, MRT- und Biopsiebefunden beruhen, eine 3- bis 6-monatige WCD-Versorgung beinhalten und nach einer erneuten Prognoseeinschätzung mit einer individualisierten Entscheidung zur dauerhaften ICD-Versorgung schließen (• Abb. 2; [10]).

\subsubsection{Valvuläre Kardiomyopathien}

In Deutschland repräsentieren die Aortenklappenstenose und die Mitralklappeninsuffizienz die am häufigsten behandelten Herzklappenvitien beim Erwach-

senen. Beide Krankheitsbilder gehen mit chronischen Volumen- und Druckbelastungen des Herzens einher und münden oftmals in einer valvulären Kardiomyopathie mit linksventrikulärer Hypertrophie und Dilatation sowie einer deutlich reduzierten LV-EF. Zumeist gelangen diese Patienten im Rahmen einer erstmaligen kardialen Dekompensation zur stationären Aufnahme, aber auch maligne Herzrhythmusstörungen, deren Risiko gerade bei hochgradigen Aortenklappenstenosen erhöht ist, führen diese zur Behandlung in die Klinik. Nach kardialer Rekompensation oder bei unmittelbarer Operationsindikation können entweder ein operativer Aortenklappenersatz (AKE), eine katheterbasierte Aortenklappenimplantation (TAVI), eine operative Mitralkappenrekonstruktion (MKR) bzw. ein Mitralklappenersatz (MKE) oder eine kathetergestützte Mitralklappenin- tervention (z. B. MitraClip ${ }^{\circledR}$, Abbott Vascular, Illinois, USA) erfolgen. Obwohl es im Anschluss mitunter bereits innerhalb kurzer Zeit zu einer deutlichen Verbesserung der kardialen Funktionsparameter kommt [40], sind zur weiteren Risikostratifikation des PHT bei Patienten mit einer $\mathrm{LV}$-EF $\leq 35 \%$ wiederholte postoperative echokardiographische Kontrollen anzuraten. Diese sollten bereits während des stationären Aufenthalts mit einem ausreichenden zeitlichen Abstand zur perioder postoperativen Katecholamingabe beginnen, einen Zwischenbefund noch vor der stationären Entlassung beinhalten und anschließend bis zur finalen Evaluation einer ICD-Indikation wiederholt werden.

Auch wenn aktuell keine ausreichenden Daten zur WCD-Verordnung nach Herzklappeneingriffen und zur weiteren Prognose der valvulären Kardiomyopathie, der LV-EF und dem Risiko eines PHT verfügbar sind, scheint es vernünftig, Herzklappenpatienten mit einer LV$\mathrm{EF} \leq 35 \%$ vorübergehend mit einem WCD zu versorgen, bis diese wieder auf über $35 \%$ angestiegen ist oder eine ICDImplantation erfolgte.

\subsection{Sekundärprophylaktische Indikation nach hämodynamisch relevanten Rhythmusstörungen}

Nachdem ein Patient eine hämodynamisch relevante, ventrikuläre Arrhythmie erlitten hat, eine reversible Ursache ausgeschlossen ist und keine Kontraindikation zur Implantation eines permanenten Defibrillators besteht, ist die sekundärprophylaktische ICD-Indikation auf Basis der geltenden Leitlinien zu stellen und eine zeitnahe ICD-Implantation durchzuführen.

Ergeben sich hingegen zeitliche Verzögerungen in der Durchführung der notwendigen Diagnostik, bestehen möglicherweise reversible Arrhythmiekausalitäten oder liegen Infektionen vor, die eine umgehende Operation verbieten, so sollte der Patient bis zum Zeitpunkt einer definitiven ICD-Imwerden (EKG-Monitor oder -Telemetrie) und ein Defibrillator zur unmittelbaren Rhythmuskonversion verfügbar sein. plantation adäquat rhythmusüberwacht 


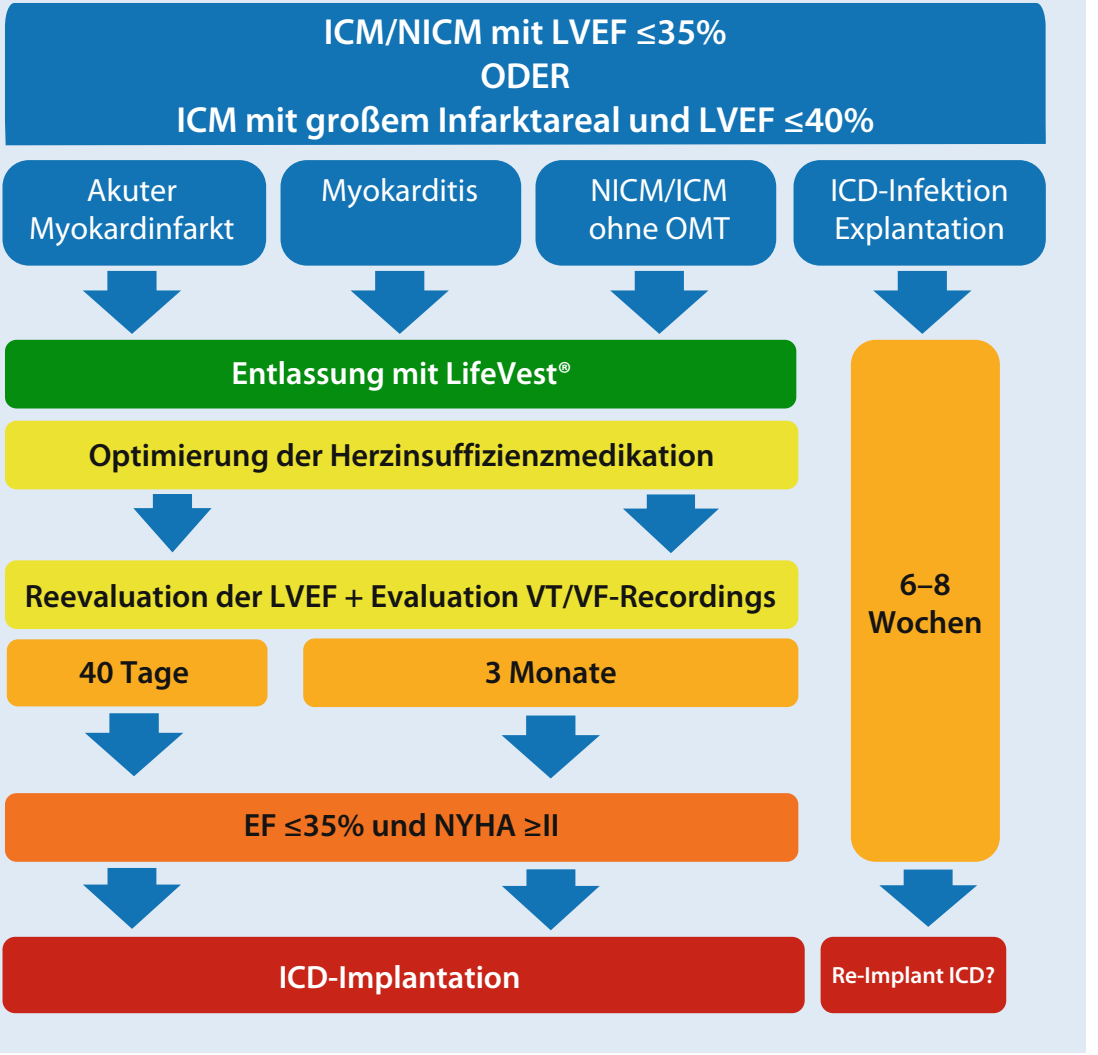

Abb. 6 ॥ Schaubild - klinisches Entlassungsmanagement. EF/LVEF linksventrikuläre Ejektionsfraktion, ICD Implantable Cardioverter-Defibrillator, ICM ischämische Kardiomyopathie, NICM nicht ischämische Kardiomyopathie, OMT ohne optimierte medikamentöse Therapie, VT ventrikuläre Tachykardie, VF ventricular fibrillation, NYHA New York Heart Assiciation Classification

Nicht immer ist allerdings eine längerfristige Hospitalisierung des Patienten sinnvoll und möglich. So erfordern beispielsweise ambulant sanierbare Wundoder Zahninfektionen zwar eine gewisse Behandlungszeit, zwingen aber nicht per se zur stationären Behandlung. Besteht in diesen Fällen dennoch ein potenziell hohes Risiko zum Auftreten maligner Arrhythmien, so kann eine überbrückende WCD-Versorgung für bis zu 3 Monate erfolgen (Klasse-IIb/CEmpfehlung, Abb. 3; [1, 2, 18]), bis die zugrunde liegende Kontraindikation beseitigt wurde und eine dauerhafte ICDImplantation erfolgen kann.

\subsection{Kontraindikation zur ICD- Implantation bei bestehender ICD- Indikation}

Besteht trotz leitliniengerechter Indikation zur ICD-Implantation eine absolute, relative oder reversible Kontraindikation,

\subsection{WCD-Indikation im Rahmen von ICD-Explantationen}

Auch wenn sich nur ein verschwindend geringer Anteil von $0,01 \%$ an perioperativen Infektionen im Rahmen des stationären Aufenthalts zur ICD-Neuimplantation in den Ergebnissen der externen stationären Qualitätssicherung in Deutschland findet, beträgt ihr Anteil doch 7,6\% der jährlichen Revisionseingriffe [41]. Somit stellt die Behandlung von "Device“-Infektionen ein relevantes Indikationsspektrum dar. Neben vorausgegangenen Aggregatwechseln oder Sondenrevisionen findet sich ihre Ursache häufig in den Implantatlangzeitfolgen, wie einer perkutanen Perforation von Systemkomponenten, bakteriellen Sondenendokarditiden unterschiedlicher Genese sowie anderen Komorbiditäten des zunehmend multimorbideren Patientenklientels. Oftmals ist dann die teilweise oder vollständige Entfernung des infizierten ICD-Systems mit Verlust des Defibrillatorschutzes unumgänglich [42-44], und erst nach der Infektionsausheilung kann, mit einigem zeitlichen Versatz, in einem zweiten operativen Schritt die erneute ICD-Implantation bzw. Ergänzung oder Reaktivierung relevanter Systemkomponenten erfolgen. Während dieser Periode ist es ratsam, eine erneute kritische Reevaluation der ICD-Indikation durchzuführen. Entsprechend den aktuellen Leitlinien [42-44] wird die erneute ICD-Implantation frühestens $72 \mathrm{~h}$ nach Bestätigung negativer Blutkulturen in Abhängigkeit von den aktuellen Infektionsparametern und dem lokalen Wundbefund empfohlen. Auf diese Weise ergibt sich zumeist eine Karenzzeit von mindestens 10 bis 14 Tagen, wobei Ellenbogen et al. klare Vorteile in einem noch längeren infektfreien Intervall sehen und im Zweifel ein protrahierteres Vorgehen empfehlen $[8,45]$. Das resultierende Zeitintervall zwischen ICD-Explantation und Reimplantation kann somit durchaus 2 bis 3 Monate betragen und sollte daher durch den Einsatz eines WCD überbrückt werden (Klasse-IIa/C-Empfehlung) [1,2, 18]. Sieht man hingegen eine Alternative in der Anlage eines subkutanen ICDs, so ist zu bedenken, dass dieses Vorge- 


\begin{tabular}{|c|c|}
\hline \multicolumn{2}{|c|}{ Abkürzungen } \\
\hline$A C E$ & „Angiotensin converting enzyme" \\
\hline AKE & Aortenklappenersatz \\
\hline$A R B$ & "Angiotensin-receptor blocker" \\
\hline ATP & Antitachykardes Pacing \\
\hline$C A B G$ & "Coronary artery bypass grafting" \\
\hline$C E$ & Communauté européenne - Europäische Gemeinschaft \\
\hline DGK & Deutsche Gesellschaft für Kardiologie - Herz- und Kreislaufforschung \\
\hline DINAMIT & The Defibrillator in Acute Myocardial Infarction Trial \\
\hline$E F$ & Ejektionsfraktion \\
\hline ESC & European Society of Cardiology \\
\hline FDA & Food and Drug Administration, USA \\
\hline$I A B P$ & Intraaortale Ballonpumpe \\
\hline$I C D$ & Implantierbarer Kardioverter/Defibrillator \\
\hline IQTIG & Institut für Qualitätssicherung und Transparenz im Gesundheitswesen \\
\hline IRIS & „Immediate Risk Stratification Improves Survival trial“ \\
\hline$L V-E F$ & Linksventrikuläre Ejektionsfraktion \\
\hline$\angle V A D$ & „Left ventricular assist device” \\
\hline MADIT & Multicenter Automatic Defibrillator Implantation Trial \\
\hline MKE & Mitralklappenersatz \\
\hline MKR & Mitralklappenrekonstruktion \\
\hline MRT & Magnetresonanztomographie \\
\hline PAVK & Peripher arterielle Verschlusskrankheit \\
\hline $\mathrm{PCl}$ & „Percutaneous coronary intervention" \\
\hline PHT & Plötzlicher Herztod \\
\hline PROLONG & $\begin{array}{l}\text { "Prolongation of Reverse remOdelling period to avoid untimely ICD impLantation in } \\
\text { newly diagnOsed heart failure usiNG the wearable cardioverter/defibrillator-Study“ }\end{array}$ \\
\hline STICH & „Surgical Treatment for Ischemic Heart Failure“ \\
\hline$T A-A K I$ & Transapikale Aortenklappenimplantation \\
\hline TAVI & „Transcatheter aortic valve implantation“ \\
\hline$T F-A K I$ & Transfemorale Aortenklappenimplantation \\
\hline VEST & Vest Prevention of Early Sudden Death Trial and Vest Registry \\
\hline$V F$ & "Ventricular fibrillation" \\
\hline VT & Ventrikuläre Tachykardie \\
\hline$W C D$ & „Wearable cardioverter/defibrillator" \\
\hline WEARIT & Wearable Defibrillator in Terminating Tachyarrhythmias Trial \\
\hline
\end{tabular}

hen im Rahmen einer Infektionsbehandlung ebenfalls mit Infektionsgefahren, einer höheren Wahrscheinlichkeit für inadäquate Schockabgaben und höheren Kosten verbunden ist $[46,47]$. (,semi-permanent pacing leads')“ perkutan über eine zentrale Vene einzubringen oder epikardiale Stimulationselektroden durch eine minimalinvasive Thorakotomie $\mathrm{zu}$ implantieren [43]. Die entsprechenden Elektroden werden dann entweder mit einem externen oder einem subkutan implantierten Schrittmacheraggregat konnektiert, welches vorübergehend die Stimulationsfunktion übernimmt. Um dennoch eine Defibrillationsmöglichkeit zu erhalten, kann dies in Kombination mit einem WCD geschehen. Allerdings sollten aufgrund der Gefahr einer Interaktion zwischen beiden Systemen eine bipolare Schrittmacherstimulation gewählt und - durch eine differenzierte Geräteprogrammierung - mögliche Geräteinteraktionen, das sogenannte „crosstalk“, ausgeschlossen werden (• Abb. 3; [48]).

\subsection{Sonderfälle}

Patienten mit einer terminalen Herzinsuffizienz auf der Warteliste zur Herztransplantation können, sofern dies die klinische Situation zulässt, mit einem WCD versorgt („bridge to transplant“) und bis zur Transplantation in das häusliche Umfeld entlassen werden (KlasseI/C-Empfehlung nach ISHLT [17] bzw. Klasse-IIa/C-Empfehlung nach ESC [2], EHRA [18] bzw. DGK-Stellungnahme [19]). Analog besteht diese Möglichkeit für Patienten mit implantiertem ,assist device“ (z.B. LVAD), die ebenfalls ein hohes Risiko zum Auftreten von lebensbedrohlichen ventrikulären Arrhythmien aufweisen (Klasse-IIa/C-Empfehlung [31]). Zwar ist in beiden Fällen auch eine dauerhafte ICD-Implantation möglich, doch ist zu bedenken, dass diese mit einer höheren Komplikationsrate verbunden sein kann [49]. Insbesondere dann, wenn im Rahmen der Transplantation nicht alle Systemkomponenten vollständig entfernt werden konnten, können sich im Langzeitverlauf ernste Probleme wie Sondenendokarditiden unter der notwendigen Immunsuppression ergeben. Zusammengefasst sollte die Systemwahl unter Abwägung der Vor- und Nachteile sowie des Tragekomforts und der voraussichtlichen Therapiedauer unter Berücksichtigung der heutzutage langen 
Transplantationswartezeiten gemeinsam mit dem Patienten gegeneinander abgewogen werden (• Abb. 4).

\section{Kosteneffektivität der temporären WCD-Therapie}

Die Kosteneffektivität einer WCD-Therapie wurde bisher in drei amerikanischen Untersuchungen $[46,47,50]$ eingehender analysiert. Vor dem Hintergrund, dass diese temporäre Therapie die stationären Behandlungskosten und die Anzahl der permanent implantierten ICD relevant reduzieren könnte, konnten Healy und Carillo eine positive KostenNutzen-Beziehung von \$15,392/QUALY im Rahmen von operativen Explantationen infizierter ICD-Systeme aufzeigen [46]. Gemeinsam ist den Studien die Einschätzung, dass die zu erwartenden Einsparungen durch die passagere WCD-Therapie die monatlichen Kosten von ca. $3000 €$ übersteigen. Somit stellt die WCD-Therapie neben einer medizinisch begründeten auch eine kosteneffektive Behandlung in der Prävention des PHT dar.

\section{Implementierung eines WCD- Programms in den klinischen Alltag}

Entscheidend für die prophylaktische Behandlung von Patienten mit einem hohen Risiko zum Auftreten lebensbedrohlicher ventrikulärer Rhythmusstörungen ist die frühzeitige Identifikation der Hochrisikopatienten. Hierzu zählen insbesondere Patienten, die an einer Kardiomyopathie mit hochgradig eingeschränkter LV-EF $(<35 \%)$ leiden und eine kardiochirurgische oder interventionelle Therapie erfahren, erstmalig eine Herzinsuffizienztherapie erhalten oder an einer Myokarditis erkrankt sind (• Abb. 5). Zu diesem Zwecke können Patienten entsprechend ihrer Herzfunktion selektioniert und differenzierten Herzrhythmus-/ Herzinsuffizienzbehandlungspfaden zugeteilt werden. Auf diese Weise ist es bereits präoperativ bzw. präinterventionell möglich, spezifische Behandlungskonzepte zur Anwendung zu bringen wie beispielsweise die prophylaktische Intraaortale-Ballonpumpe (IABP) zur kardialen
Unterstützung bei Patienten mit einer ischämischen Kardiomyopathie, die präoperative Levosimendanapplikation bei valvulären Kardiomyopathien oder die prophylaktische WCD-Versorgung zum Schutz vor dem PHT während der präoperativen Wartezeit. Entsprechend differenziert kann dann auch die postoperative Nachsorge in Abhängigkeit von der LV-EF, der Schwere der Herzinsuffizienz und der Prognose geplant werden (• Abb. 6). Neben einer angemessenen medikamentösen Herzinsuffizienztherapie sollten diese Hochrisikopatienten das Akutkrankenhaus dann auch durch einen WCD geschützt verlassen und in eine ambulante oder stationäre Rehabilitationsmaßnahme überwiesen werden. Etwa 90 Tage nach dem Eingriff sind dann die aktuelle LV-EF und der klinische Schweregrad der Herzinsuffizienz (NYHA-Klasse) in einer fachübergreifenden Ambulanz im Herzrhythmus-/ Herzinsuffizienzteam erneut zu bestimmen und zu bewerten. Zeigt sich hierbei ein klinisch deutlich verbesserter Patient mit einer LV-EF von mehr als 35\%, ist die Implantation eines ICD nur noch in Einzelfällen indiziert. Hingegen rechtfertigen eine unverändert hochgradig eingeschränkte oder gar verschlechterte LV-EF ( $\leq 35 \%)$ und eine NYHA-Klasse $\geq$ II die umgehende ICD-Implantation zu diesem Zeitpunkt.

\section{Schlussfolgerung}

Der WCD stellt eine effektive und sichere zusätzliche Option, einerseits in der Behandlung von Patienten mit einem vorübergehend erhöhten Risiko für den PHT sowie andererseits in der passageren Behandlung von Patienten mit einem dauerhaft erhöhten Risiko für maligne Arrhythmien dar, falls sich vorübergehend ein permanentes ICDSystem verbietet. Auf diese Weise steht ein erweitertes Zeitfenster zur Evaluation einer dauerhaften ICD-Indikation zur Verfügung, indem Patienten mit einer hochgradig eingeschränkten LV-EF, einer erstmalig diagnostizierten Herzinsuffizienz oder nach Revaskularisationsoder Herzklappeneingriffen eine angemessene ventrikuläre Erholungsphase eingeräumt wird. Somit kann sich die An- zahl der primärprophylaktischen ICDImplantationen um die Anzahl reduzieren, die sich bisher nach einer ventrikulären Restitution als nicht mehr indiziert erweisen. Zum gegenwärtigen Zeitpunkt existieren, trotz fehlender randomisierter prospektiver Studien ausreichend große Registerdaten, die die Funktionalität und Wirksamkeit des WCDs belegen. Diese Tatsache war Grundlage zur Therapieempfehlung in internationalen Leitlinien, und so stellt der WCD auch insbesondere für herzchirurgische Patienten eine medizinisch sinnvolle und patientenorientierte Behandlungsoption dar.

\section{Korrespondenzadresse

Dr. H. Burger
Abteilung für Herzchirurgie,
Kerckhoff-Klinik GmbH
Benekestraße 2-8, 61231 Bad
Nauheim, Deutschland
h.burger@kerckhoff-klinik.de

\section{Einhaltung ethischer Richtlinien}

Interessenkonflikt. H. Burger ist Mitglied der Deutschen Gesellschaft für Thorax-, Herz- und Gefäßchirurgie (DGTHG), Mitglied im Bund Deutscher Chirurgen (BDC), Stellv. Vorsitzender der Arbeitsgruppe „Herzrhythmusstörungen“ der DGTHG, Mitglied des „expert panel“ HSM/ICD des Instituts zur Qualitätssicherung und Transparenz im Gesundheitswesen (IQTIG, Berlin), zuvor im Institut für angewandte Qualitätsförderung und Forschung im Gesundheitswesen (AQUA, Göttingen) und Leiter des Workshopzentrums „Elektrodenextraktionen" der Fa. Spectranetics/Philips an der Kerckhoff-Klinik in Bad Nauheim. Beziehungen zu teils honorierten Lehr-, Vortrags- und Beratungszwecken bestehen mit folgenden Firmen und Fachgesellschaften: Abbott-Medical/St. Jude Medical, Boston Scientific, Biotronik, Cook Medical, Medtronic Medical, Sorin Group, Spectranetics Medical/Philips, Zoll Medical, Deutsche Gesellschaft für Thorax-, Herzund Gefäßchirurgie (DGTHG), Deutsche Gesellschaft für Kardiologie - Herz- und Kreislaufforschung (DGK) und Deutsche Gesellschaft für Kardiotechnik (DGfK). J. Schmitt: Beziehungen zu teils honorierten Lehr-, Vortrags- und Beratungszwecken bestehen mit folgenden Firmen und Fachgesellschaften: Biotronik, CVRx, Medtronic, Fa. Zoll und St. Jude Medical/Abbott M. Knaut ist Mitglied der Deutschen Gesellschaft für Thorax-, Herz- und Gefäßchirurgie (DGTHG), Mitglied im Bund Deutscher Chirurgen (BDC) und Mitglied der Deutschen Gesellschaft für Kardiologie - Herz und Kreislaufforschung, Mitglied der Arbeitsgruppe "Herzrhythmusstörungen". Beziehungen zu teils honorierten Lehr-, Vortrags- und Beratungszwecken bestehen mit folgenden Firmen und Fachgesellschaften: Boston Scientific, Medtronic Medical, Spectranetics Medical/Philips, CVRx und Zoll Medical. T. Eitz ist Mitglied der Deutschen Gesellschaft für Thorax-, 
Herz- und Gefäßchirurgie (DGTHG) und Mitglied der Arbeitsgruppe "Herzrhythmusstörungen" der DGTHG. Beziehungen zu teils honorierten Lehr-, Vortrags- und Beratungszwecken bestehen mit folgenden Firmen und Fachgesellschaften: Abbott-Medical/St. Jude Medical, Spectranetics Medical/Philips, Biotronik SE \& Co. KG und Deutsche Gesellschaft für Kardiologie - Herz- und Kreislaufforschung (DGK). C. T. Starck ist Mitglied der Deutschen Gesellschaft für Thorax-, Herzund Gefäßchirurgie (DGTHG), 2. Beisitzer des Vorstandes der Arbeitsgruppe „Herzrhythmusstörungen“ der DGTHG, Fellow der European Heart Rhythm Association (FEHRA) und Mitglied des Technical Advisory Board der Firma Biotronik. Beziehungen zu teils honorierten Lehr-, Vortrags- und Beratungszwecken bestehen mit folgenden Firmen und Fachgesellschaften: Biotronik, Boston Scientific, Gook Medical, Medtronic, Angiodynamics, Sorin Group und Spectranetics/ Philips. S. Hakmi ist Mitglied der Deutschen Gesellschaft für Thorax-, Herz- und Gefäßchirurgie (DGTHG), Mitglied der Deutschen Gesellschaft für Kardiologie (DGK), Mitglied der Arbeitsgruppe "Herzrhythmusstörungen“ und Leiter des Workshopzentrums „Elektrodenextraktionen" der Fa. Spectranetics/Philips an der Klinik für Herz- und Gefäßchirurgie am UHZ. Beziehungen zu teils honorierten Lehr-, Vortrags- und Beratungszwecken bestehen mit folgenden Firmen und Fachgesellschaften: Spectranetics/Philips IGT Devices, Zoll Medical und Deutsche Gesellschaft für Kardiologie - Herz- und Kreislaufforschung (DGK). A. Siebel ist Mitglied der Deutschen Gesellschaft für Thorax-, Herzund Gefäßchirurgie (DGTHG) und Vorsitzender der Arbeitsgruppe "Herzrhythmusstörungen" der DGTHG. Beziehungen zu teils honorierten Lehr-, Vortrags- und Beratungszwecken bestehen mit folgenden Firmen und Fachgesellschaften: Abbott-Medical/St. Jude Medical, Medtronic Medical, Spectranetics Medical/ Philips, Deutsche Gesellschaft für Thorax-, Herz- und Gefäßchirurgie (DGTHG) und Deutsche Gesellschaft für Kardiologie - Herz- und Kreislaufforschung (DGK). A. Böning: Beziehungen zu teils honorierten Lehr-, Vortrags-und Beratungszwecken bestehen mit folgenden Firmen und Fachgesellschaften: Zoll Medical.

Dieser Beitrag beinhaltet keine von den Autoren durchgeführten Studien an Menschen oder Tieren.

Open Access. Dieser Artikel wird unter der Creative Commons Namensnennung 4.0 International Lizenz (http://creativecommons.org/licenses/by/4.0/deed. de) veröffentlicht, welche die Nutzung, Vervielfältigung, Bearbeitung, Verbreitung und Wiedergabe in jeglichem Medium und Format erlaubt, sofern Sie den/die ursprünglichen Autor(en) und die Quelle ordnungsgemäßnennen, einen Link zur Creative Commons Lizenz beifügen und angeben, ob Änderungen vorgenommen wurden.

\section{Literatur}

1. DenekeT,Borggrefe M, Hindricks G, KirchhofP, Kuck KH, Stellbrink C, Eckardt L (2017) Kommentar zu den ESC-Leitlinien 2015 „Ventrikuläre Arrhythmien und Prävention des plötzlichen Herztodes". Kardiologe 11:27-43

2. Priori SG et al (2015) 2015 ESC Guidelines for the management of patients with ventricular arrhythmias and the prevention of SCD. Eur Heart J 36(41):2793-2867

3. Verma $A$, Wulffhart $Z$, Lakkireddy $D$, Khaykin $Y$, Kaplan A, Sarak B, Biria M, Pillarisetti J, Bhat P, Di Biase $L$ et al (2010) Incidence of left ventricular function improvement after primary prevention
ICD implantation for non-ischaemic dilated cardiomyopathy: a multicentre experience. Heart 96:510-515

4. Solomon SD et al (2005) Sudden death in patient with myocardial infarction and left ventricular dysfunction, heart failure, or both. N Engl J Med 353(7):744

5. Hohnloser SH et al (2004) Prophylactic use of an implantable cardioverter-defibrillator after acute myocardial infarction (DINAMIT). N Engl J Med 351(24):2481-2488

6. Steinbeck G et al (2009) Defibrillator Implantation Early after Myocardial Infarction (IRIS). NEngI J Med 361:1427-1436

7. Van der Heijden AC, Borleffs CJ, Buiten MS, Thijssen J, van Rees JB, Cannegieter SC, Schalij MJ, van Erven $L$ (2015) The clinical course of patients with implantable defibrillators: extended experience on clinical outcome, device replacements, and device-related complications. Heart Rhythm 12:1169-1176

8. Wäßnig Netal(2016) Experience with the wearable cardioverter-defibrillator in patients at high risk for sudden cardiac death. Circulation 134(9):635-643

9. Epstein AE et al (2013) Wearable cardioverterdefibrillator use in patients perceived to be at high risk early post myocardial infarction. JACC 62:2000-2007

10. Kutyifa V et al (2015) Use of the wearable cardioverter defibrillator in high-risk cardiac patients: data from the prospective registry of patients using the wearable cardioverter defibrillator (WEARIT-II registry). Circulation 132(17):1613-1619

11. Feldman AM et al (2004) Use of a wearable defibrillator in terminating tachyarrhythmias in patients at high risk for sudden death: results of WEARIT/BIROAD. Pacing Clin Electrophysiol 27:4-9

12. Chung MK et al (2010) Aggregate national experience with the wearable cardioverterdefibrillator, event rates, compliance, and survival. J Am Coll Cardiol 56:194-203

13. Zishiri ET et al (2013) Early risk of mortality after coronary artery revascularization in patients with left ventricular dysfunction and potential role of the wearable cardioverter defibrillator. Circ Arrhythm Electrophysiol 6(1):117-128

14. Duncker D, Haghikia A, König T, Hohmann S, Gutleben KJ, Westenfeld R, Oswald H, Klein $\mathrm{H}$, Bauersachs J, Hilfiker-Kleiner D, Veltmann C (2014) Risk for ventricular fibrillation in peripartum cardiomyopathy with severely reduced left ventricular function-value of the wearable cardioverter/ defibrillator. Eur J Heart Fail 16:1331-1336

15. Duncker D, König T, Hohmann S, Bauersachs J, Veltmann C (2017) Avoiding untimely Implantable cardioverter/defibrillator implantation by intensified heart failure therapy optimization supported by the wearable cardioverter/defibrillator-the PROLONG study. J Am Heart Assoc 6(1):e4512

16. Duncker D, König T, Hohmann S, Bauersachs J, Veltmann C (2017) Ventricular arrhythmias in patients with newly diagnosed nonischemic cardiomyopathy: insights from the PROLONG study. Clin Cardiol 40(8):586-590

17. Gronda E et al (2006) Heart rhythm considerations in heart transplant candidates and considerations for ventricular assist devices: International Society for Heart and Lung Transplantation guidelines for the care of cardiac transplant candidates-2006. J Heart Lung Transplant 25:1043-1056

18. Reek $S$ et al (2017) The wearable cardioverterdefibrillator: current technology and evolving in- dications. EHRA Scientific Documents Committee. Europace 19(3):335-345

19. Schwab JO, Bänsch D, Israel C, Nowak B (2015) Stellungnahme zum Einsatz des tragbaren Kardioverter/Defibrillators. Kardiologe 9:165-170

20. El-Chami M et al (2012) Ventricular arrhythmia after cardiac surgery - incidence, predictors, and outcomes. J Am Coll Cardiol 60(25):2664-2671

21. Al-Khatib SM et al (2011) Non-Evidence-Based ICD Implantations in the United States. JAMA 305(1):43-49

22. Kirkfeldt RE et al (2014) Complications after cardiac implantable electronic device implantations: an analysis of a complete, nationwide cohort in Denmark. Eur Heart J 35:1186-1194

23. Greenberg H et al (2004) Analysis of mortality events in the multicenter automatic defibrillator implantation trial (MADIT II). J Am Coll Cardiol 43(8):1459-1465

24. Broessner $\mathrm{G}$ et al (2012) Update on therapeutic temperature management. Crit Care 16(Suppl 2):A1

25. Rao MP et al (2017) Sudden cardiac death in patients with Ischemic heart failure undergoing coronary artery bypass grafting: results from the surgical treatmentfor ischemicheartfailure $/ \mathrm{STICH}$ randomized clinical trial. Circulation. https://doi. org/10.1161/CIRCULATIONAHA.116.026075

26. Haxhibeqiri-Karabolic I (2014) Improvement of ejection fraction after coronary artery bypass grafting surgery in patients with impaired left ventricularfunction. Med Arch 68(5):332-334

27. Solomon SD et al (2001) Recovery of ventricular function after myocardial infarction in the reperfusion era: The healing and early afterload reducing therapy study. Ann Intern Med 134(6):451-458

28. Sutton M (2003) Left ventricular remodeling and ventricular arrythmias after myocardial infarction. Circulation 107(20):2577-2582

29. Bhatt AS et al (2017) Adverse remodeling and reverse remodeling after myocardial infarction. Curr Cardiol Rep 19:71

30. Vakil K, Florea V, Koene R, Kealhofer JV, Anand I, Adabag S (2016) Effect of coronary artery bypass grafting on left ventricular ejection fraction in men eligible for implantable-cardioverter defibrillator. Am J Cardiol 117(6):957-960

31. Piccini JP et al (2016) AHA science advisory wearable cardioverter-defibrillator therapy for prevention of sudden cardiac death. Circulation 133(17):1715-1727

32. Windecker S et al (2014) ESC/EACTS Guidelines on myocardial revascularization. Eur Heart J 35:2541-2619

33. Køber L et al (2016) Defibrillator implantation in patients with nonischemic systolic heart failure, DANISH. N Engl J Med 375:1221-1230

34. Konstam MA, Efficacy of Vasopressin Antagonism in Heart Failure Outcome Study With Tolvaptan (EVEREST) Investigators et al (2007) Effects of oral tolvaptan in patients hospitalized for worsening heart failure - the EVEREST outcome trial. JAMA 197(12):1319. https://doi.org/10.1001/jama.297. 12.1319

35. Ponikowski P et al (2016) ESC Guidelines for the diagnosis and treatment of acute and chronic heart failure. The Task Force for the diagnosis and treatment of acute and chronic heart failure of the European Society of Cardiology (ESC). Developed with the special contribution of the Heart Failure Association (HFA) of the ESC. Eur Heart J37(27):2129-2200

36. Kao AC et al (2012) Wearable defibrillator use in heart failure investigators. Wearable defibrillator 
use in heart failure: results of a prospektive registry. BMC Cardiovasc Disord 12:123

37. Duncker D, Westenfeld R, Konrad T, Pfeffer $T$, Correis de Freitas C, Pfister R, Thomas D, Fürnkranz A, Andrié R, Napp A, Schmitt J, Karolyi L, Wakili $\mathrm{R}$, Hilfiker-Kleiner D, Bauersachs J, Veltmann CG (2017) Elevated risk for life-threatening arrhythmia in newly diagnosted peripartum cardiomyopathy-Data from a German multicentre study. Clin Res Cardiol 106(Suppl1). https:// doi.org/10.1007/s00392-017-1105-2

38. Konrad T, Sonnenschein S, Schulz E, Wenzel P, Wagner S, Schmidt KH, Münzel T, Theis C, Rostock T, Mollnau H (2016) What is the incidence of ventricular arrhythmias in the mid-term phase of acute myocarditis? New insights from the wearable cardioverter/defibrillator. Clin Res Cardiol 105(Suppl 1). https://doi.org/10.1007/ s00392-016-0967-z

39. Kindermann l et al (2008) Predictors in outcome in patients with suspected myocarditis. Circulation 118:639-648

40. Schmidt T, Frerker C (2017) Ergebnisse der transkatheter Aortenklappenimplantation (TAVI) bei Patienten mit einer stark reduzierten linksventriklulärer Ejektionsfraktion $\leq 20 \%$. Pressetext DGK 04/2017

41. IQTIG - Institut für Qualitätssicherung und Transparenz im Gesundheitswesen (2017) Qualitätsreport 2016. Spree Druck, Berlin

42. WilkoffBL et al (2009) Transvenous lead extraction: HRS expert consensus on facilities, training, indications, and patient management. Heart Rhythm 6(7):1085-1104

43. Kusumoto FM et al (2017) HRS expert consensus statement on cardiovascular Implantable electronic device lead management and extraction. Heart Rhythm:31080-31089. https://doi.org/10.1016/j. hrthm.2017.09.001

44. Carillo R et al (2010) HRS expert consensus on transvenous lead extractions and AHA statement on ICD infection and management. Circulation 121:458-477

45. Ellenbogen K et al (2016) Benefit of the wearable cardioverter defibrillator in protecting patients after Implantable ICD-Cardioverter defibrillator explant. JACC Clin Electrophysiol. https://doi.org/ 10.1016/j.jacep.2016.09.002

46. Healy CA, Carillo RG (2015) Wearable cardioverterdefibrillator for prevention of sudden cardiac death after infected implantable cardioverterdefibrillator removal. A cost-effective evaluation. Heart Rhythm 12:1565-1573

47. Quast et al (2017) Outpatient treatment with the wearable cardioverter defibrillator: clinical experience in two Dutch centres. Neth Heart J 25:312-317

48. Schmitt J, Abaci G, Johnson V, Erkapic D, Gemein C, Chasan R, Weipert K, Hamm CW, Klein HU (2017) Safety of the wearable cardioverter defibrillator (WCD) in patients with implanted pacemakers. Pacing Clin Electrophysiol 40(3):271-277

49. Pettit SJ, Petrie MC, Connelly DT, Japp AG, Payne JR, Haj-Yahia S et al (2012) Use of implantable cardioverter defibrillators in patients with left ventricular assist devices. Eur J Heart Fail 14:696-702

50. Sanders GD, Owens DK, Hlatky MA (2015) Potential cost-effectiveness of wearable cardioverterdefibrillator early after myocardial infarction. J Innov Cardiac Rhythm Manag 6:1929-1940

51. Zimarino M, Ricci F, Romanello M et al (2016) Complete myocardial revascularization confers a larger clinical benefit when performed with state-of-the-art techniques in high-risk patients with multivessel coronary artery disease: a metaanalysis of randomized and observational studies. Catheter Cardiovasc Interv 87(1):3-12

52. Nombela-Franco L, Mitroi CD, Fernández-Lozano I et al (2012) Ventricular arrhythmias among implantable cardioverter-defibrillator recipients for primary prevention: impact of chronic total coronary occlusion (VACTO Primary Study). Circ Arrhythm Electrophysiol 5(1):147-154

53. Olgin JE (2018) Vest prevention of early sudden death trial-VEST [annual ACC congress, Orlando/USA 10.03.2018]. http://www.acc.org/latestin-cardiology/clinical-trials/2018/03/09/08/06/ vest.Zugegriffen: 9. Apr. 2018
Autoren WERKSTATT

GRATIS

Ein Service für Autoren von Springer Medizin

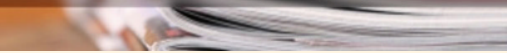

Fortbildungen für Autoren und Gutachter

Die ersten Veröffentlichungen sind für jeden Mediziner ein wichtiger Schritt in seiner Karriere als Wissenschaftler. Wissenschaftliche Artikel sind entscheidend dafür, dass die eigene Arbeit in der Community wahrgenommen wird. Es geht darum, die eigenen Ideen national und international auszutauschen und sicherzustellen, dass die Ergebnisse Wirkung erzielen. Die Online-Kurse der Autorenwerkstatt helfen, sich leicht einen Überblick über das Schreiben, Einreichen, Begutachten und Veröffentlichen eines Manuskripts zu verschaffen.

5 Online-Kurse zu den wichtigsten Standards des wissenschaftlichen Publizierens:

- Wie verfasse ich ein Manuskript?

- Writing in English für deutschsprachige Autoren

- Wie funktionieren Publikation und Begutachtung?

- Anleitung zur Open-AccessVeröffentlichung

- Leitfaden zur Peer-ReviewBegutachtung

Für alle, die auf SpringerMedizin.de registriert sind!

Jetzt gratis fortbilden unter www.springermedizin.de/ autorenwerkstatt/ 
Hier steht eine Anzeige.

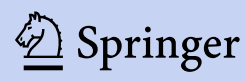

